\title{
EVENTS AND EVENT PROCESSES
}

\author{
Tadeusz KRUPA \\ Faculty of Management \\ Warsaw University of Technology, 02-524 Warszawa, Poland \\ email: T.Krupa@wz.pw.edu.pl
}

\begin{abstract}
Presented article describes the issues known from the logical structures characterization theory [2] used in this case to describe the events designed for the needs of proper functioning of organizational structures. Necessary formalization is used strictly for the maintenance of the concept discipline. Event theory [5] is used in the designing of complex structures of organizational systems for which the basic necessity is its compliance with the set functional description. Final organization's characteristics are determined by many factors, such as: a) completeness and non-contradiction of the functional specification b) theoretical correctness of the functional transformation of the organization into the design of its organizational structure c) correctness of the technical project realization.
\end{abstract}

Key words: object, object configuration, events on the object channels, symbolism and operations on events, regular expression of events, event loops, process as a sequence of events, event net, relations and conflicts in event nets, graph figures in event nets, net correctness.

\section{Introduction}

Event theory includes a wide research scope that formulates assumptions, hypothesis and proof describing the essence and relations of events. This paper is limited to the research area, in which a single event is defined as the change of the state of the distinguished element, which is called a channel, with the assumption of the finite number of identifiable channels and finite scope of identifiable states of every channel and in conditions where in given time the channel is in one and only one of its states, listed in the scope of states for this particular channel, and also where the time necessary to switch channels is negligible.

Event theory, through its assumptions and inference rules, can be used to define and execute two tasks:

- assurance of the completeness and non-contradiction of the functional specification of designed organizational structure,

- assurance of theoretical correctness of the functional transformation of the organization into the theoretically correct design of its organizational structure construction.

Basic concepts of this theory are: objects, object channels, channel states and events in channels, sequences of series, alternative, synchronous and cyclical events, event nets and areas, forbidden and obligatory event graph figures, regular event network, event network canonical correctness.
In event theory channels are considered as relations between objects that communicate with each other (see Fig. 1 and Fig. 2), with an assumption that every outgoing channel of the object is its belonging channel (on these channel procedures for given object determine the states, which become accessible for the procedures of object's environment).

Every channel that belongs to a given object, represents, in particular time, one of the distinguished states of this object as its own state. Many channels can belong to a single object. Object procedures perform the state forcing operation on these channels.

Let us assume that two objects $0_{i}, 0_{j}$ with unknown internal structure (see Fig. 2) communicate through the $\mathrm{K}$ channel set. Any $\mathrm{K}_{\mathrm{p}} \in \mathrm{K}$ channel can be in one of its $\mathrm{S}_{\mathrm{v}} \in \mathrm{S}$ admissible states in any time. The communication of objects is based on the fact that the equivalent $K_{i}, K_{j}$ channel subsets, where $K_{i} \cap K_{j}=\varnothing$, determine (marked: !) the $\mathrm{S}_{\mathrm{v}}$ states according to their internal functioning procedures and simultaneously in "suitable" time they "observe" (marked: ?) the channels of their neighbor. This observing usually means the control of $\mathrm{O}_{\mathrm{i}}$ object (adequately $\mathrm{O}_{\mathrm{j}}$ ) over selected $\mathrm{K}_{\mathrm{j}}$ (adequately $\mathrm{K}_{\mathrm{i}}$ ) channels.

Such type of object communication is called loose (autonomic) - contrary to the enforced communication, present in logical control systems. The following part of the deliberations in the article considers the autonomic communication between the $\mathrm{O}_{\mathrm{i}}, \mathrm{O}_{\mathrm{j}}$ objects. 


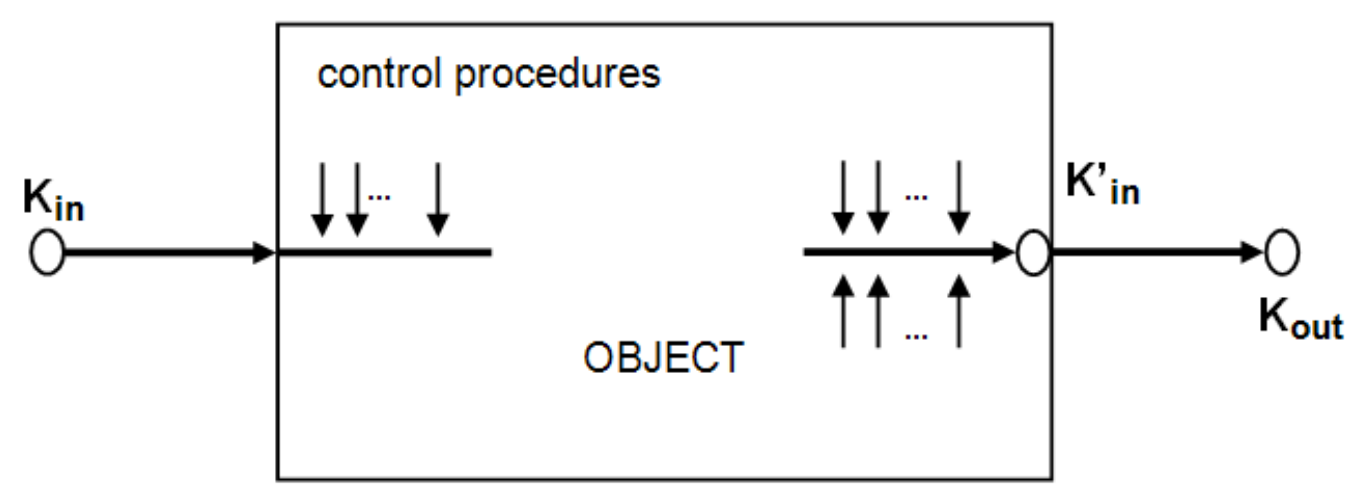

Figure 1. Outgouing $\mathrm{K}_{\text {out }}$ and incoming $\mathrm{K}_{\text {in }}$ belonging channels

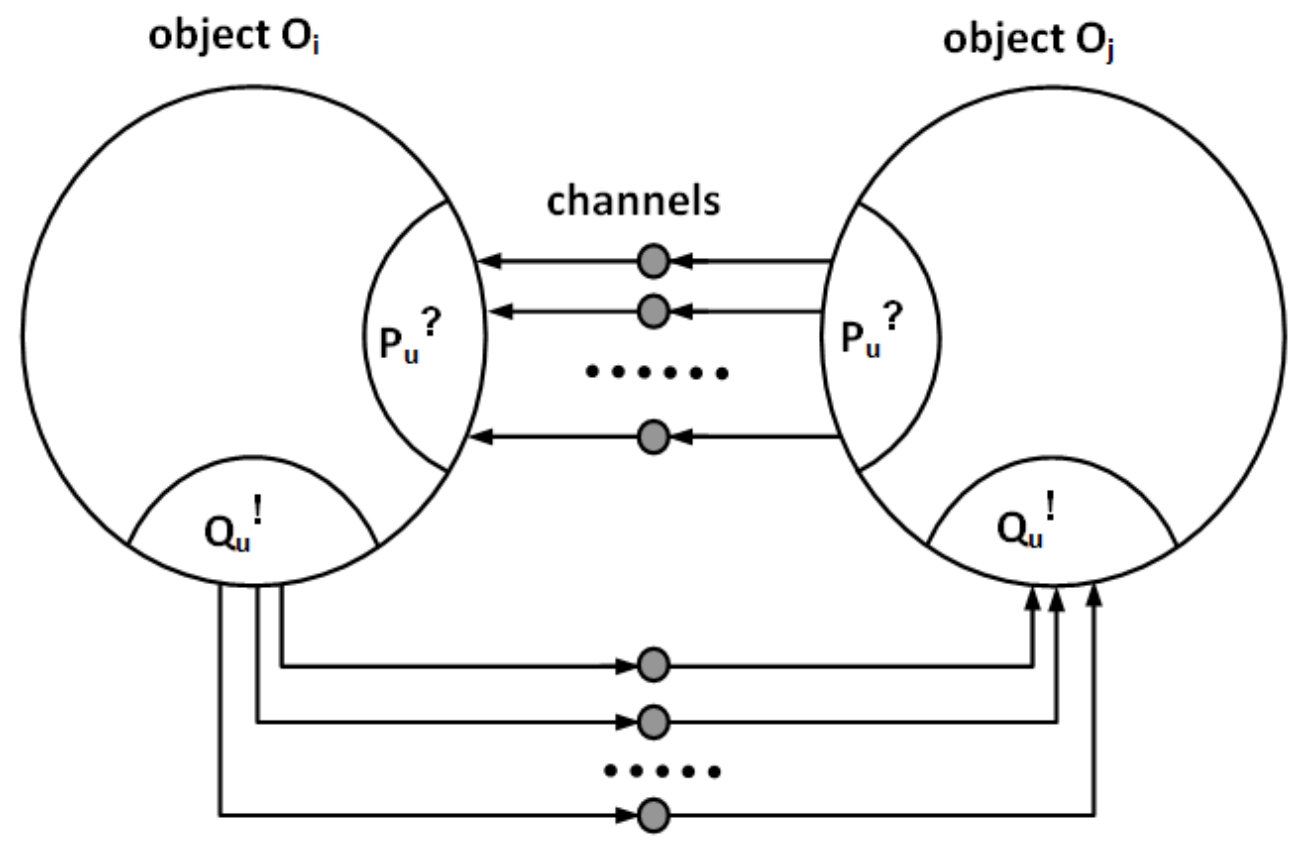

Figure 2. Relations between communicating objects.

Belonging outgoing channels and incoming channels of $\mathrm{O}_{i}, \mathrm{O}_{j}$ objects

Series of channel state change events are realized during the communication of $\mathrm{O}_{\mathrm{i}}, \mathrm{O}_{\mathrm{j}}$ objects on the $\mathrm{K}_{\mathrm{i}}, \mathrm{K}_{\mathrm{j}}$ channels (see Fig. 2). Channel state changes create the process observed by the $\mathrm{O}_{\mathrm{i}}, \mathrm{O}_{\mathrm{j}}$ objects.

Fig. 3 presents the configuration of objects that create a superior object. This is an object whose incoming and outgoing channels (channels $\mathrm{K}_{1,0}, \mathrm{~K}_{1,5}$ ) are the channels connecting internal objects (objects: $\mathrm{O}_{2}$, $\mathrm{O}_{4}$ ) with the environment. The proper outgoing channel of an object can be an incoming channel of one or several objects' that belong to a configuration or an outgoing channel of this configuration.

Object interpretation (modeling) of the organizational system's reality can be performed in a way that the whole internal memory of the organization will be described with the configuration of internal objects and incoming and outgoing channels of these objects. In general case object configuration can have a multilevel structure, where objects with the same or different configuration communicate through channels. 


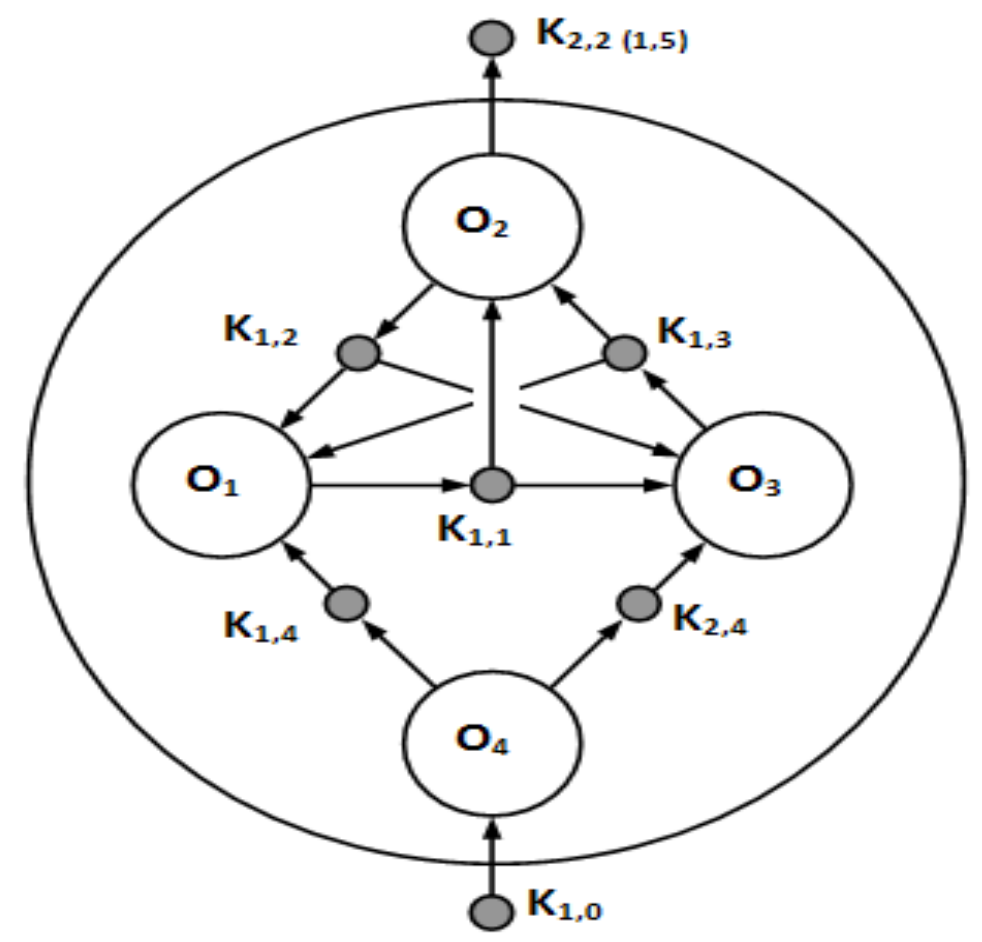

Figure 3. Multiplexing channels of communicating objects. Superior object as a configuration of internal objects

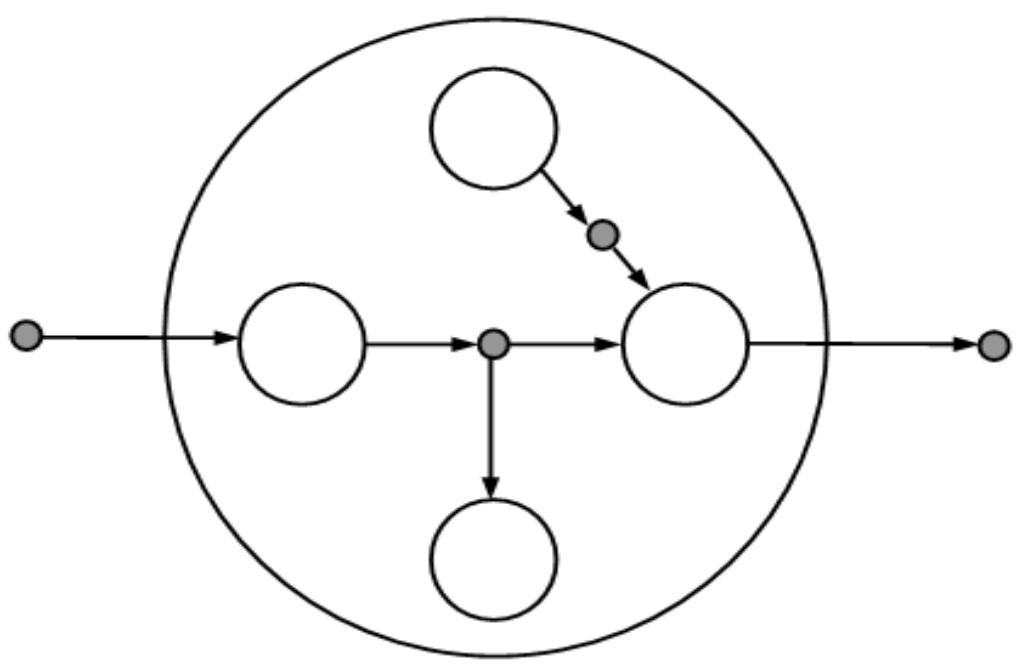

Figure 4. Example of object configuration

Objects create object configuration in the form of a superior object when every incoming and outgoing channel of this object is at the same time a incoming or outgoing channel of a single internal object of this configuration.

At least one incoming and one outgoing channel presence must be assumed for the existence of an object.
At least one incoming and one outgoing channel as well as the presence of two internal objects of this configuration must be assumed for the existence of the object configuration in the form of a superior object. Example of object configuration is presented in the Fig. 4. 
Objects and channels are connected in any configuration adequately through objects and channels. Fig. 4 presents example of two objects connected in a configuration with one channel (outgoing and incoming).

\section{Event and channel concepts}

In real life events are usually connected with the consequences of a fact, which can be observed and measured. The causes of events are not always obvious. For the deliberations on event in real life it is necessary to determine their objective area.

Deterministic events, for which the cause and effect chain is known in particular area, and random (probabilistic) events, which causes are beyond the objective area, can occur in a synonymously determined objective area.

Examples of deterministic events are: change of traffic lights in communication, loading a fork-lift transporter in a marked space near a machine, freeing financial resources due to liquidation of endangered bank loan portfolio. Examples of random events are: initiation of fire protection alarm, chemical reactor cooling system breakdown, slump at stock market.

Event is modeled as a change of the state of particular distinguished channel of an object. Object and its channels represent, in the objective area, expected fragments of reality. Every occurrence mechanisms can be either simple (e.g. the change of traffic lights) or extremely complex (e.g. slump at stock market).

In the presented approach object is represented with a collection of belonging channels (also known as outgoing channels), with the assumption that each belonging channel can be described with finite scope of its states. If in deliberations one would assume that a database file will be treated as a channel and its records as the allowable states of this channel, therefore the database file needs to be represented not by a record but rather by one of the finite number of classes that can include this record. The record cannot be interpreted as a channel state because one cannot effectively operate with all of its executions.

Including the record into one of the possible classes should always guarantee a synonymous answer (classes should be disjointed in the set of all possible executions of records and their number should be redefined).

Example of a single event is the change of the outgoing state in the object's channel (see Fig. 5) caused by the forcing operation performed by an object, to which the given channel belongs. Single event is always connected with the emerging of a new state from a particular scope of states for given channel. New channel state is started at the moment of the succession of the event.

Single event will be marked with the $Z_{\mathrm{i}}$ symbol and the event set as $Z$. Events form the $Z$ set that happen successively create the event sequence.

At given moment of time the channel is able to remember only one state that belongs to the scope of allowable states of this channel.

Event marked with a single symbol $Z_{\mathrm{i}}$ is called an elementary event. Event marked with at least two symbols of elementary events, connected with an operation symbol indicating a connection between these events, is called a composed event.

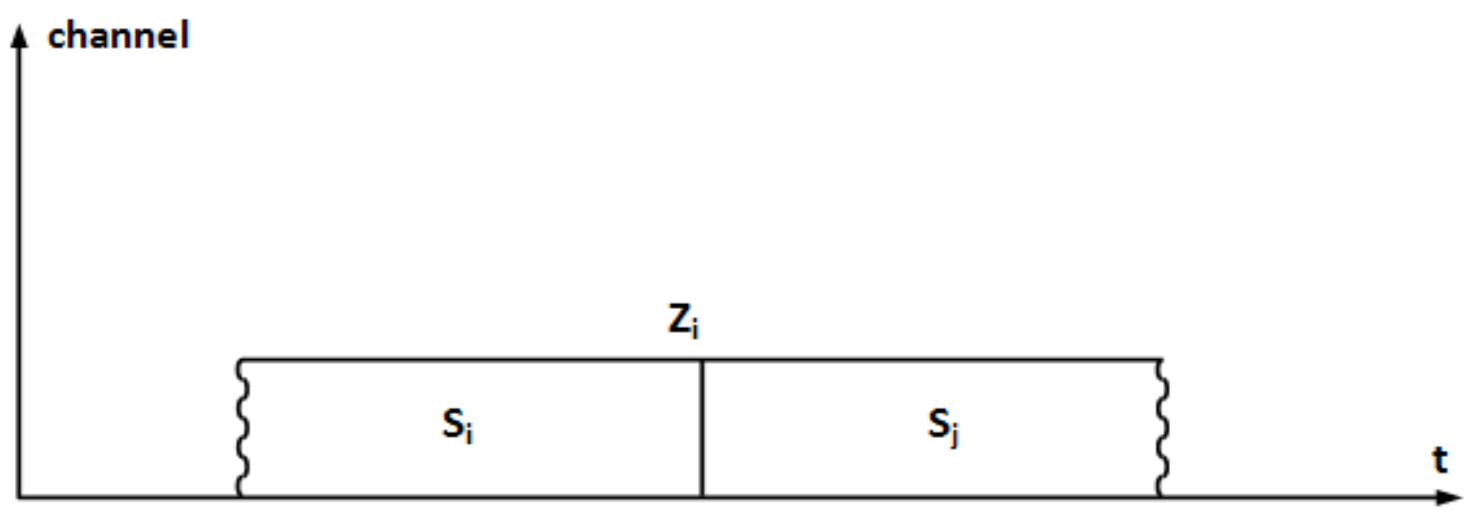

Figure 5. Event $\mathbf{Z}_{\mathbf{i}}$ and $\mathbf{S}_{\mathbf{i}}, \mathbf{S}_{\mathbf{j}}$ states in the channel 
In order to simplify the deliberations and recording the event symbols will also be interpreted as the symbols of the channel forced states. This assumption is based on the fact that with every event the channel creates a new state corresponding with this event. With this assumption the scope of channel states mutually synonymously responds to the scope of allowable events on given channel.

Two elementary events $Z_{i}, Z_{j}$ will be called the alternative (disjunctive) events in case when one of the events excludes the happening of the second event. Alternative events will be connected with the disjunction operation symbol " $\vee$ "in the form of the following expression:

$$
Z_{i} \vee Z_{j}
$$

or in the form of expression:

$$
Z_{1} \vee Z_{2} \vee \ldots \vee Z_{m}
$$

in case of $m$ alternative events.

We assume that due to the assurance of the event operation correctness, the alternative events of given sequence of alternative events will always precede one event realized on channel other than the event belonging to the alternative events sequence.

Elementary events $Z_{i}, Z_{j}$ will be called the synchronous events in case, when they happen in given time interval independent of each other so that none of the events is not an effect of another event. Synchronous events will be connected with the "\#" synchronism operation symbol and recorded in the form of the following expression:

$$
\mathrm{Z}_{1} \# \mathrm{Z}_{2}
$$

or in the form of expression:

$$
\mathrm{Z}_{1} \# \mathrm{Z}_{2} \# \ldots \# \mathrm{Z}_{\mathrm{m}}
$$

in case of $m$ alternative events.

We assume that due to the assurance of the event operation correctness, the synchronous events of given sequence of events will always relate to the change of states taking place at different channels.

Sequences of synchronous events, similarly to the sequences of alternative events, always precede one event realized on channel other than the channels of alternative events sequences.

Synchronous events are also called as parallel or conjunctive.
Elementary events $Z_{i}, Z_{j}$ will be called the series events in case, when the direct and necessary condition of $Z_{j}$ event happening is the happening of the $Z_{i}$ event. Event series will be connected with the " $\wedge$ " synchronism operation symbol and recorded in the form of the following expression:

$$
Z_{i} \wedge Z_{j}
$$

or in the form of expression:

$$
Z_{1} \wedge Z_{2} \wedge \ldots \wedge Z_{m}
$$

in case of $m$ alternative events.

We assume that due to the assurance of the event operation correctness, the event series of given sequence of events will always relate to the change of states taking place at different channels for any pair of the events form this sequence.

Elementary event $Z_{\mathrm{i}}$ will be called a cyclical (iterative) event in case, when it does not happen or happens creating a sequence of event series.

Cyclical events will be marked with the "*" cycle operation symbol and recorded in the following form:

$$
\mathrm{Z}_{\mathrm{i}}{ }^{*}
$$

According to the definition of the cyclical event it is expressed as:

$$
Z_{i}^{*}=Z_{\phi} \wedge Z_{i} \wedge Z_{i} \wedge \ldots
$$

or in more detailed form

$$
\mathrm{Z}_{\mathrm{i}}^{*}=\mathrm{Z}_{\phi} \vee\left[\mathrm{Z}_{\mathrm{i}} \wedge \mathrm{Z}_{\mathrm{i}}^{*}\right]
$$

where: $Z_{\phi}$ - empty (fictional) event.

According to the definition the cyclical event relates to the same channel.

Events created as a result of connection of events with elementary operations $\vee, \#, \wedge$ and $*$ are called regular events.

Brackets determine the sequence of the $\vee, \#, \wedge, *$ operation interpretation in composed events.

Operations $\vee, \#, \wedge,{ }^{*}$ on the $\mathrm{Z}$ event set determine the alternative $(\vee)$, synchronous $(\#)$, series $(\wedge)$ cyclical $(*)$ event relations.

\section{Examples of event sequences}

Examples of compound events with short interpretation comment are presented below.

Example: $\quad Z_{1} \#\left[Z_{2} * \wedge Z_{3}{ }^{*}\right]$ 
Synchronous with $Z_{1}$ event two cyclical events are realized $Z_{2}{ }^{*}$ and next (after finishing of $Z_{2}{ }^{*}$ ) event $Z_{3}{ }^{*}$.

Event graphs are the graphical interpretation of events and regular expressions. Fig. 6 presents the graph of events in a regular expression with the following form

Example:

$$
\left[Z_{1} \wedge\left[\left[\left[Z_{2} \#\left[Z_{3} \wedge Z_{4}\right]\right] \vee\left[Z_{5} * \# Z_{6}\right]\right]\right] \wedge Z_{7}\right] \vee Z_{8} *
$$

Arcs of the graph, with assigned synchronous events, are connected with the marking of an angle.

In order to assure the synonymous nature of the event graph interpretation in the Fig. 6 fictional (empty) events were introduced $Z_{\phi 1}-Z_{\phi 4}$ in relation to the outgoing regular expression. Assurance of the synonymy of the symbolic recording interpretation with relation to the graph form is a necessary condition for the proper construction of process models.

It is important to mention that $Z_{2}$ and $Z_{4}$ events are not synchronous, similarly to $Z_{6}$ and $Z_{\phi 4}$ events. The following couples of events can be the $<Z_{2}, Z_{3}>$ and $<\mathrm{Z}_{6}, \mathrm{Z}_{\phi 3}>$ couples.

Event graph vertexes shown in the Fig.6 connect the events not indicating the relations between them. In most cases these relations can be correctly interpreted with the analysis of the event graph, although this type of activity does not guarantee the synonymy in case of an attempt of defining the correctness of the event graph. For this purpose event dispersion and concentration constraints, for alternative and synchronous events, are laid upon the graph vertexes. As a result the graph vertexes are changed with dispersing and concentrating knots of alternative and synchronous events.

Series and cyclical events do not have to be distinguished in order to assure the synonymy of the event sequence interpretation. During the transformation of vertexes into knots, vertexes that connect more than one preceding event with following event are changed with two knots first knot is concentrating and the second one is dispersing. One of the particular events of vertex transformation, connecting series events with cyclical events, into concentrating and alternative knots is presented in the Fig. 7.

Introduction of concentrating knot $\mathrm{V}^{>}$and dispersing $\mathrm{V}^{<}$ led to the necessity of introduction of a fictional (empty) event $Z_{\phi}$ '. In this new situation the cyclical event $Z_{5}{ }^{*}$ will be replaced with complex event $Z_{\phi}{ }^{\prime} \wedge\left(Z_{5} \wedge Z_{\phi}{ }^{\prime}\right)^{*}$. It is easy to spot that both expressions are equivalent in relation to produced events:

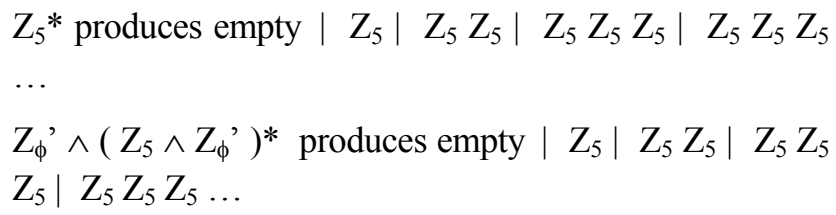
$\cdots$

$Z_{\phi}{ }^{\prime} \wedge\left(Z_{5} \wedge Z_{\phi}{ }^{\prime}\right)^{*}$ produces empty $\left|Z_{5}\right| Z_{5} Z_{5} \mid Z_{5} Z_{5}$ $Z_{5} \mid Z_{5} Z_{5} Z_{5} \ldots$

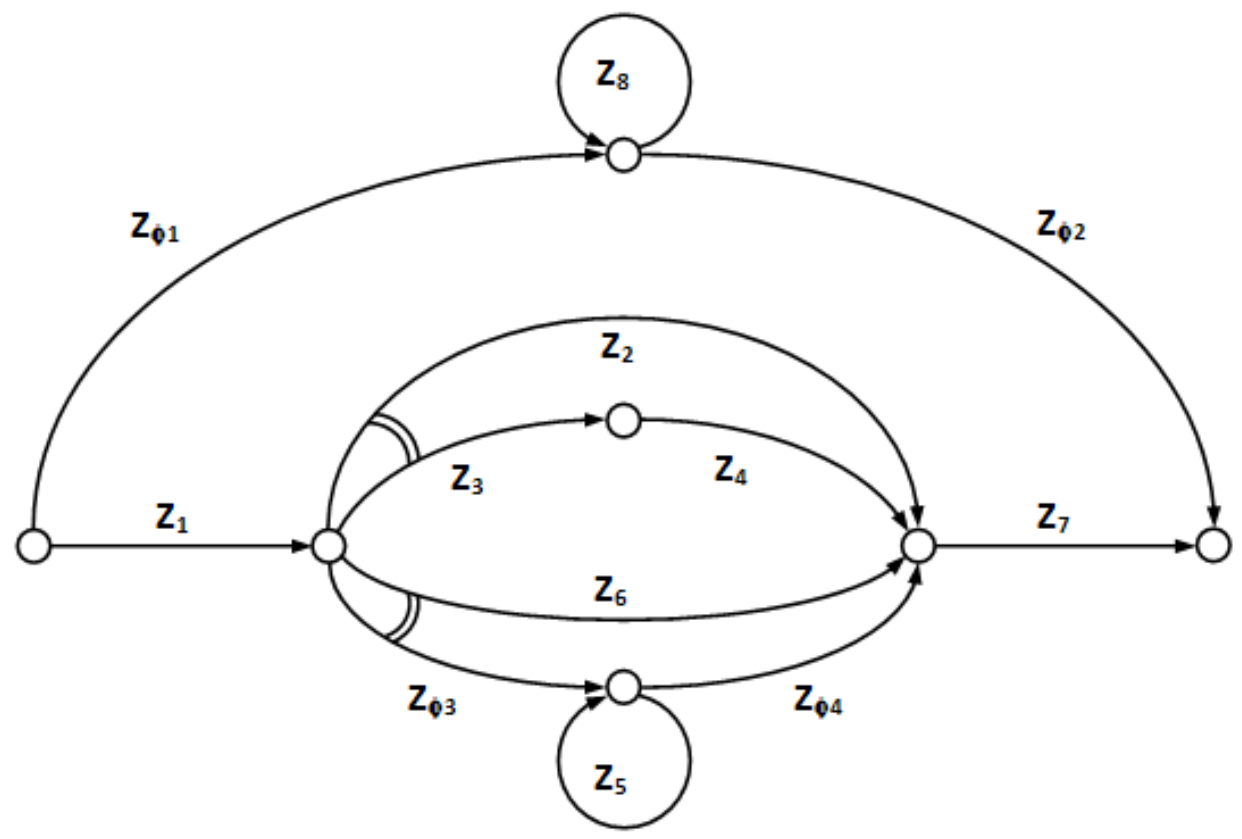

Figure 6. Event graph for regular expression 

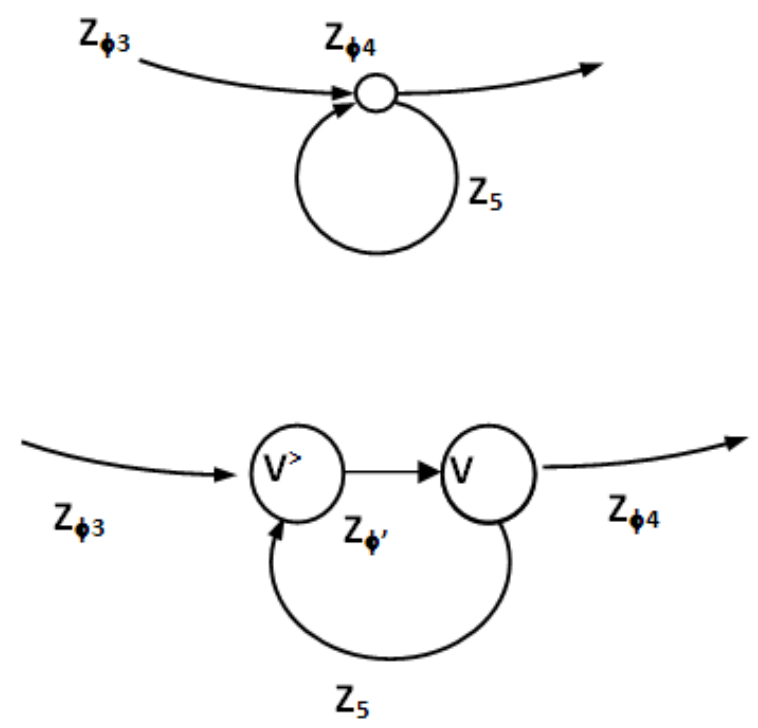

Figure 7. Transformation of vertex in to a concentrating knot

\section{$4 \quad$ Event knots}

Alternative dispersive event knot connects one and only one event preceding event with one of many other following alternative events (see Fig. 8). If only one event takes place one speaks of a pair of series events - in such case the symbol $\mathrm{V}$ is not used.

The condition of proper $\mathrm{V}^{<}, \mathrm{V}^{>}$knot functioning is the assurance of alternative activities for both dispersion and concentration of events. In the second case the condition of $Z_{j}$ event occurrence is the presence of one and only one event from the $Z_{1}, Z_{2}, \ldots, Z_{\mathrm{m}}$ event set.
It is important to mention that only the $Z_{1}, Z_{2}, \ldots, Z_{m}$ events originating directly from the $\mathrm{V}^{<}$alternative connection are the alternative events.

Synchronous knot of dispersing events connects one and only one preceding event with many other synchronous events (see Fig. 9). If only one event takes place one speaks of a pair of series events - in such case the \# symbol is not used.

Synchronous knot of concentrating events connects many synchronous events with one and only one following event. If the preceding event consists of only one event one speaks of a pair of series events - in such case the \# symbol is not used.
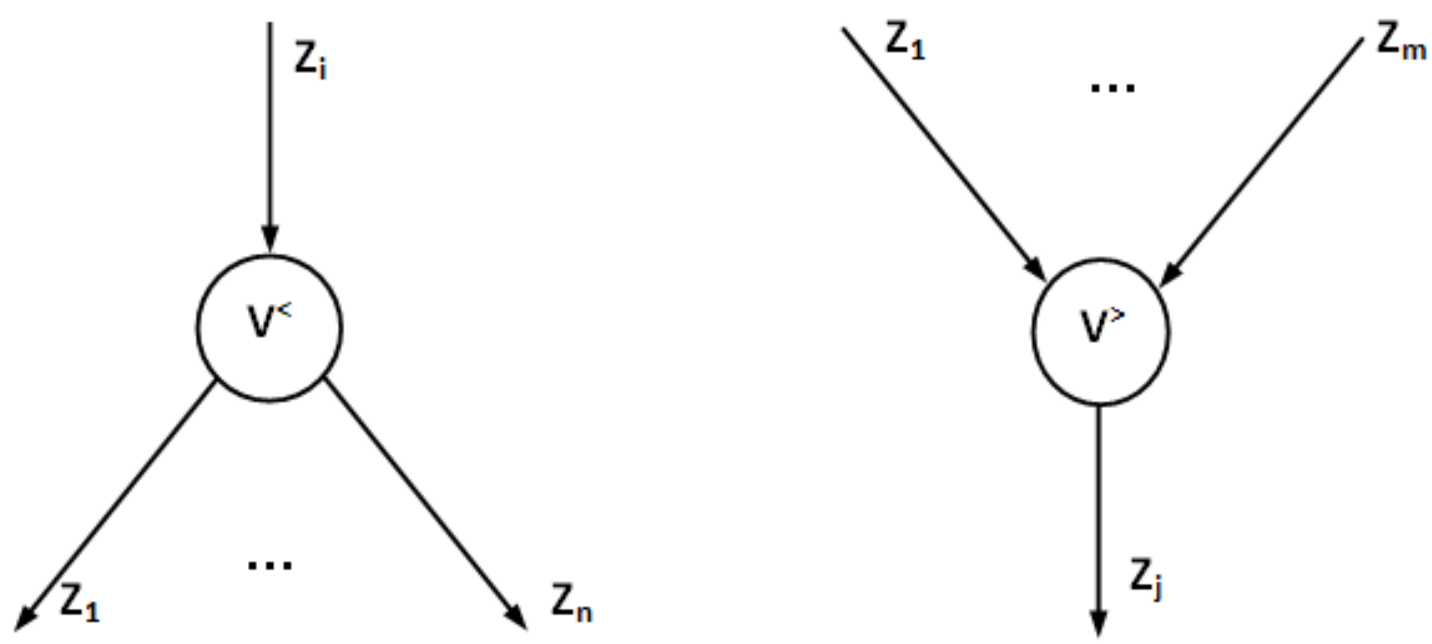

Figure 8. Dispersing and concentrating alternative event knots 

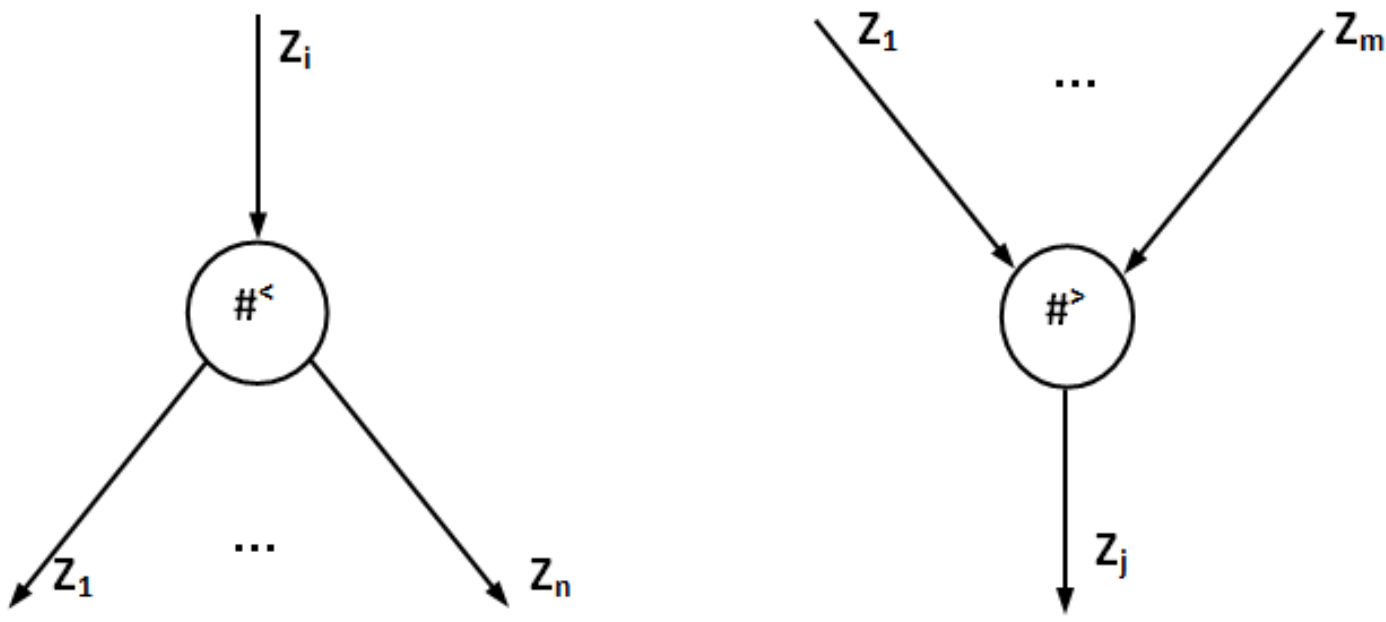

Figure 9. Synchronous knots of concentrating and dispersing events

The condition of proper $\#^{<}, \#^{>}$knot functioning is the assurance of synchrony of activities for both dispersion and concentration of events. In the second case the condition of $Z_{j}$ event occurrence is the presence of one and only one event from the $Z_{1}, Z_{2}, \ldots, Z_{\mathrm{m}}$ event set.

It is important to mention that only the $Z_{1}, Z_{2}, \ldots, Z_{m}$ events originating directly from the $\#^{<}$synchronous connection are the synchronous events.

\section{$5 \quad$ Event sequences}

Series event sequence takes place in a situation when the occurrence of following event is conditioned with the occurrence of preceding event. Fig. 10 presents a sequence of four events $Z_{a}, Z_{b}, Z_{c}$ and $Z_{d}$ that can be presented as symbols as follows:

$$
Z_{\mathrm{a}} \wedge Z_{\mathrm{b}} \wedge Z_{\mathrm{c}} \wedge Z_{\mathrm{d}}
$$

$Z_{d}$ event happens only if it is preceded by the following $Z_{\mathrm{a}}, Z_{\mathrm{b}}$ and $Z_{\mathrm{c}}$ events.

Alternative series event sequence takes place in a situation when after alternative event dispersing knot $\mathrm{V}^{<}$at least two sequences of series events occur.

Example of two alternative series event sequences can be recorded in the following way:

$$
\left[Z_{\mathrm{a}} \wedge Z_{\mathrm{b}} \wedge Z_{\mathrm{c}} \wedge Z_{\mathrm{d}}\right] \vee\left[Z_{\mathrm{e}} \wedge Z_{\mathrm{f}} \wedge Z_{\mathrm{g}} \wedge Z_{\mathrm{h}}\right]
$$

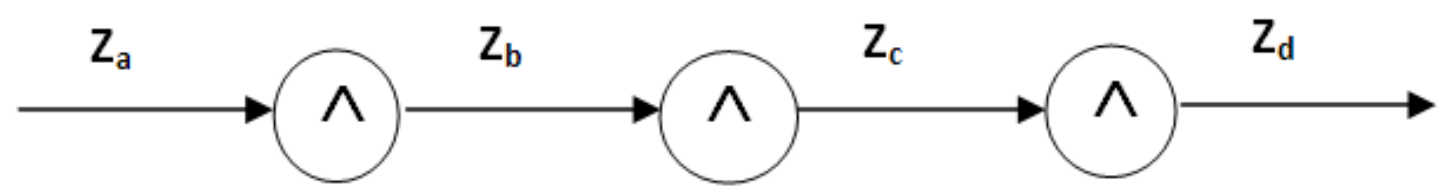

Figure 10. Example of series event sequence 


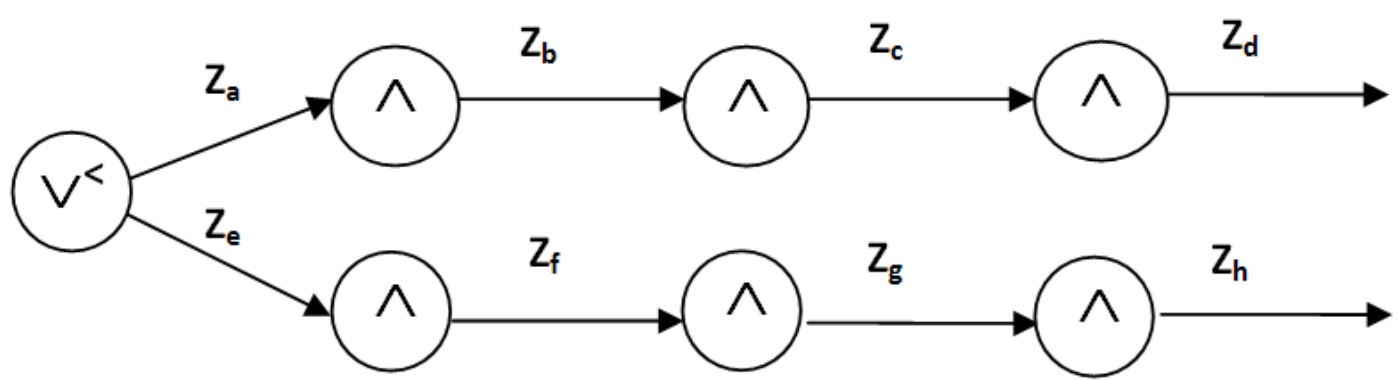

Figure 11. Example of alternative series event sequences

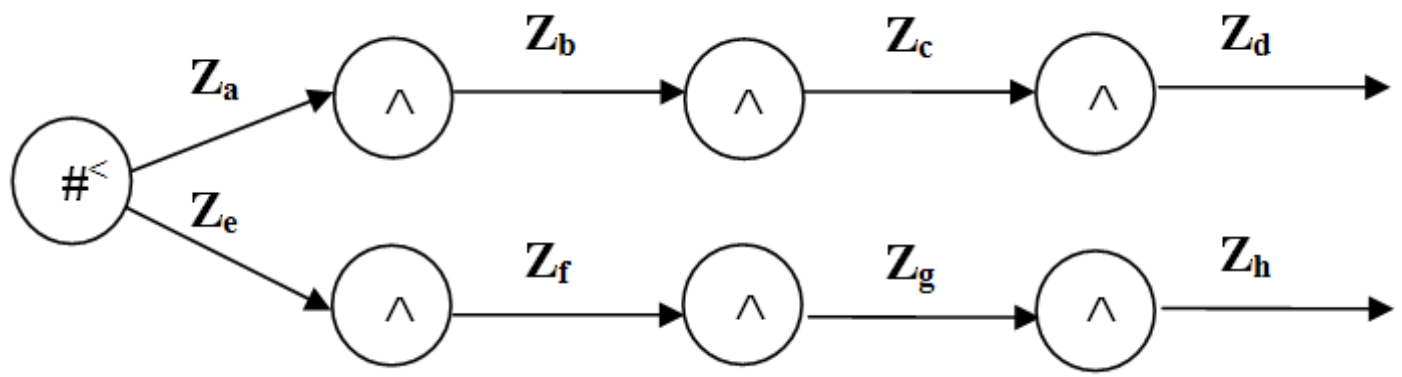

Figure12. Example of synchronous series event sequences

From both series sequences events from only one of them occur (see Fig. 11).

The condition of proper alternative series event sequences functioning is the assurance of alternativeness of activities for both dispersion and concentration of events. In the second case all alternative series event sequences should be ended with $\mathrm{V}^{>}$concentrating knot of alternative events.

Synchronous series event sequence takes place in a situation when after synchronous event dispersing knot $\#^{<}$at least two sequences of series events occur. Example of two synchronous series event sequences can be recorded in the following way:

$$
\left[Z_{\mathrm{a}} \wedge Z_{\mathrm{b}} \wedge Z_{\mathrm{c}} \wedge Z_{\mathrm{d}}\right] \#\left[Z_{\mathrm{e}} \wedge Z_{\mathrm{f}} \wedge Z_{\mathrm{g}} \wedge Z_{\mathrm{h}}\right]
$$

From both series sequences events from both of them occur (see Fig. 12).

The condition of proper synchronous series event sequences functioning is the assurance of synchrony of activities for both dispersion and concentration of events. In the second case all synchronous series event sequences should be ended with $\#^{\ngtr}$ concentrating knot of synchronous events.

Cyclical event sequence takes place in a situation when performances of given series sequence, closed in $(\ldots)^{*}$ brackets, are compliant with the definition of cyclical event presented above.

after alternative event dispersing knot $\mathrm{V}^{<}$at least two sequences of series events occur.

Figure 13 presents the example of cyclical event sequence recorded in the following way:

$$
\left(Z_{\mathrm{a}} \wedge \mathrm{Z}_{\mathrm{b}} \wedge \mathrm{Z}_{\mathrm{c}}\right)^{*}
$$

possible executions of this sequence are:

$Z_{\phi}$ - empty event (lack of event)

$$
\begin{aligned}
& Z_{a} \wedge Z_{b} \wedge Z_{c} \\
& Z_{a} \wedge Z_{b} \wedge Z_{c} \wedge Z_{a} \wedge Z_{b} \wedge Z_{c} \\
& Z_{a} \wedge Z_{b} \wedge Z_{c} \wedge Z_{a} \wedge Z_{b} \wedge Z_{c} \wedge Z_{a} \wedge Z_{b} \wedge Z_{c}
\end{aligned}
$$

Similarly to the cyclical series events, alternative cyclical events are also possible:

$$
\left(Z_{1} \vee Z_{2} \vee \ldots \vee Z_{m}\right)^{*}
$$

and cyclical synchronous

$$
\left(Z_{1} \# Z_{2} \# \ldots \# Z_{m}\right)^{*}
$$

Their analysis and transformations are omitted in further deliberations. Detailed description of event operation properties is a part of event algebra. 


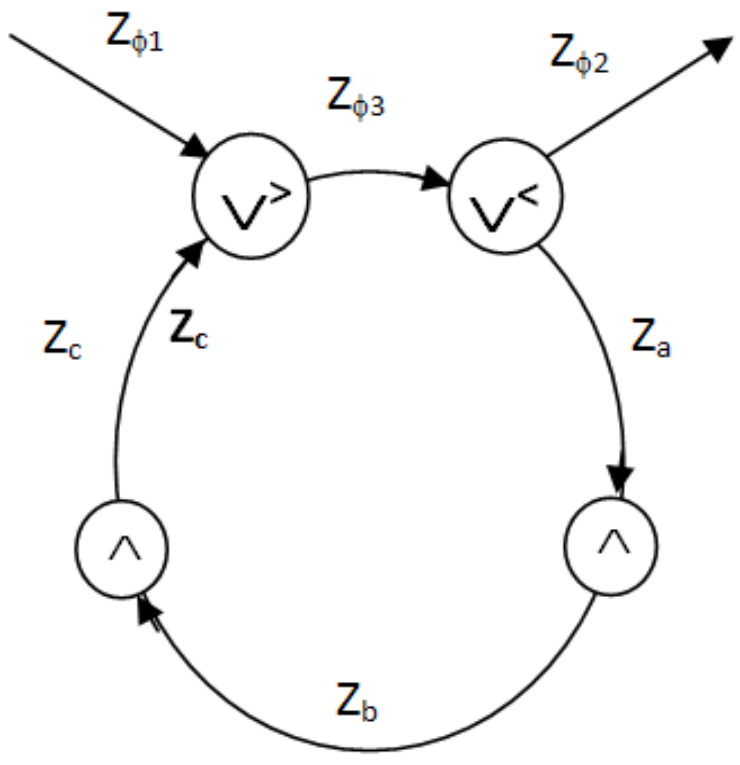

Figure 13. Example of cyclical event sequence

6

\section{Event nets}

Event net is constructed from:

- series event sequences,

- alternative series event sequences,

- synchronous series event sequences,

- cyclical event sequences.

Event net is a model of event processes. Its structure determines the execution of the processes, although the set of different process executions in case of cyclical events presence is theoretically a set of infinite size. All executions (processes) of the event net can be successfully presented if cyclical event sequences do not occur in the event net.

Event net is a model of event processes, which allows describing the processes that are correct from the point of view of their realization. Definition and the correctness conditions of event processes in each case should be specified in the categories that guarantee the verification (validation) of the correctness of their performance.

Figure 14 presents an example of event net with cyclical events sequences, alternative series event sequences and synchronous series event sequences, constructed on the basis of the regular expression event graph, presented in the Figure 6.
Processes in properly constructed event net (or in other words the executions in event net) should flow in a way that assures:

- possibility to perform every event and every event sequence in the net at least once,

- event net have one and only one distinguished starting event $Z_{\mathrm{A}}$ preceding the execution of all other events and one and only one finishing event $Z_{B}$ that takes place after execution of all events that could be executed,

- starting event $Z_{\mathrm{A}}$ can be performed only in case when none of the other events in the net is not and cannot be executed in the net,

- finishing event $Z_{B}$ in event net can only be executed as the last event process of this net.

Conclusions presented above indicate that event net can be deliberated in two following situations:

- net is not active - none of the events from the event net processes is executed and the net is waiting for the execution of the $\mathrm{Z}_{\mathrm{A}}$ starting event,

- net is active - events from the event net processes are executed and the net is waiting for the execution of the $Z_{B}$ finishing.

Event process is executed in the net through some of the cyclical event sequences and alternative series event sequences and within them through all areas of synchronous series event sequences. 


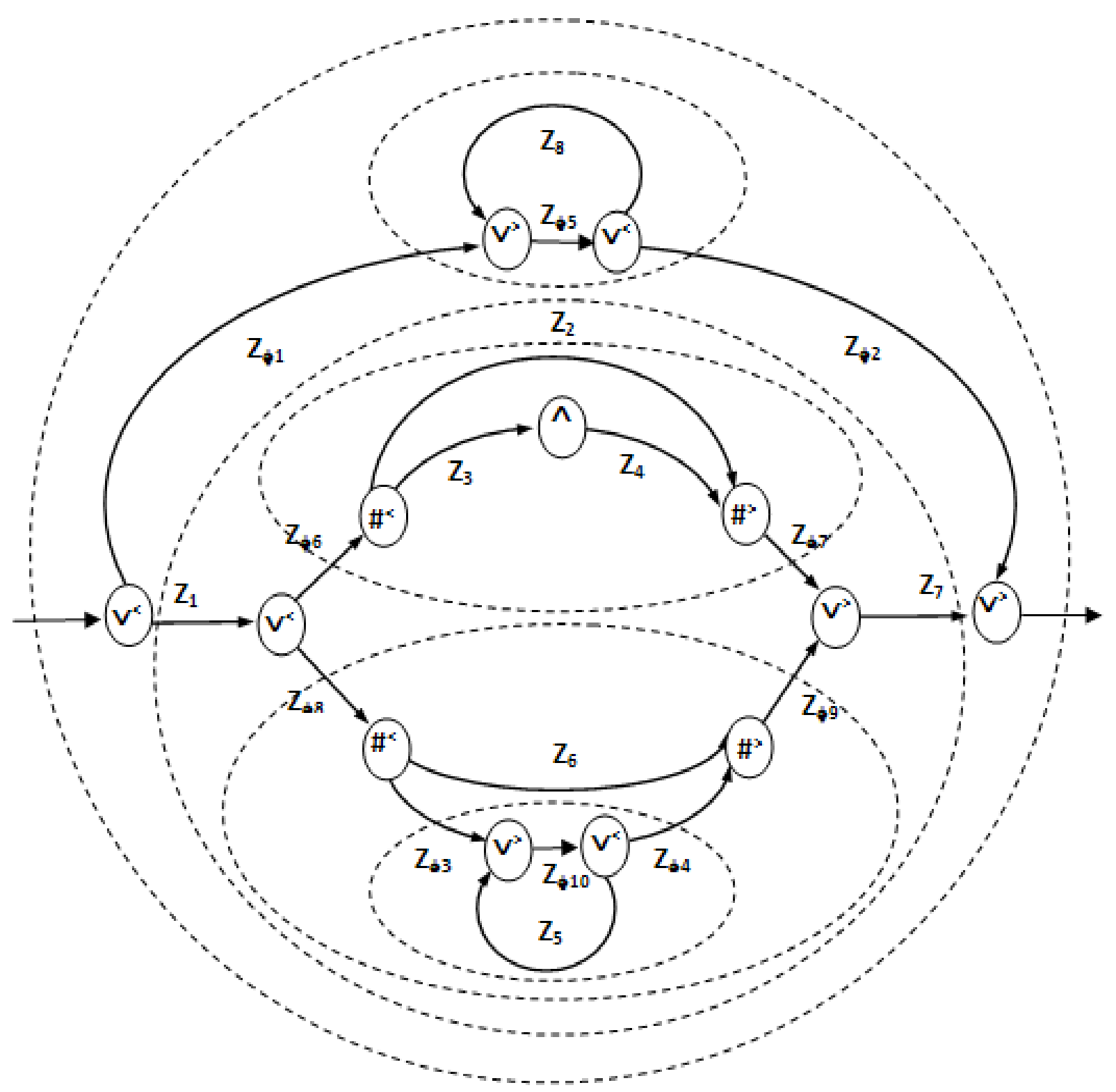

Figure 14. Example of event net

Event net area with one and only one distinguished starting event and one and only one distinguished finishing event will be called the event subnet.

The illustration of event subnet of the event net presented in the Fig. 14 is the fragments marked with the dotted line (all of six possible event subnets are presented in the figure).

Main feature of such defined event subnet is the fact that it represents one event that consists of many internal events of this subnet.
Correct event net is a structure in which other subnets can be presented in the form of a single event representing the whole outgoing net. This informal expression requires a more detailed description and comments on the possible net transformations.

Presented statement leads to the structural method of activity leading to the achievement of correct network.

Structural method of proper event net also assumes that:

- every process has one distinguished start and end,

- processes can cluster in self for any depth, 
- processes in the same depth connect with knots (dispersing and concentrating) connecting their starts and ends in a way that indicates the character of the relations between processes.

\section{Time lapse, conflicts and relations on event nets}

Time lapse is an essential characteristic of all physical processes. Logical connections of the processes and secondly the time conditionings are present in many deliberations on process modeling.

It is necessary to determine the coordinates and time lapse units in order to observe the process flow in time (time clocks of the modeled system).

Time counting needs to consider the relations between time clocks in particular subnets in case of net decomposition into event subnet areas.

Time in event nets flows , in waiting” for the happening of next events. The assumption is that the time of the event, understood as the change of state, is negligible. Event net is a scheme according to which new events occur after given time.

Occurrence of events must synonymously relate to the net structure. Therefore the event net presented in Fig. 14 generates, for example, the following allowable event productions (sequences):

empty $\left|Z_{8}\right| Z_{8} Z_{8}|\ldots| Z_{1}\left[Z_{3} Z_{4}\right] \#\left[Z_{2}\right] Z_{7} \mid \ldots$

Sequences of alternative series events should start and finish with dispersing and concentrating alternative knots. Proper structural rules need to be complied in order to assure these conditions during the construction of the net.

Rules result from relations, which can occur between any two events in the event net structure. Analysis of possible event relations indicates that if some of them occur in certain configurations with other relations, they can become the cause of erroneous event processes.

Alternative events, according to the definition (symbolic recording or event net), should not appear synchronously (e.g. at the same time) in alternative concentrating knots.

Synchronous conflict if alternative events should be prevented during the event net designing phase.
Elimination of conflicts through tests can be extremely time-consuming and difficult, when the net has already been constructed.

Synchronous series event sequences should start and end with dispersing and concentrating synchronous knots.

According to the definition (symbolic or event net) synchronous events should not occur alternatively in synchronous concentrating knots.

Alternative conflict of synchronous events should be prevented during the event net designing phase. Elimination of conflicts through tests, similarly to previous case, can be extremely time-consuming and difficult, when the net has already been constructed.

Tolerating alternative conflicts of synchronous events leads to the "pursuit of events" what equals to the loss of consistency between net structure and its functioning (event net stops being the process model).

Connections consisting of events between any pair of events $Z_{k}, Z_{1}$ in the net are called the event paths and marked as $Z_{k} \rightarrow Z_{1}$ or in a shorter form as $Z_{k} \rightarrow$, if a series of events leads from $Z_{k}$ event to $Z_{1}$ event.

If the path $Z_{\mathrm{k} \rightarrow 1}$ leads from event $Z_{\mathrm{k}}$ to event $Z_{1}$ and the $Z_{1 \rightarrow k}$ path leads form $Z_{1}$ event to $Z_{k}$ event than these paths create a cyclical path marked as $Z_{k \leftrightarrow 1}$ for any $Z_{x}$, $Z_{\mathrm{y}}$ event pair on this path.

Two events $Z_{i}, Z_{j}$ with a common starting or finishing knot in an event net with single distinguished starting event $Z_{A}$ and single distinguished finishing event $Z_{B}$, where the events $Z_{i}, Z_{j} \neq Z_{A}, Z_{B}$, can create four special cases, of concentrating $>$ and dispersing $<$ knots and events related with them, in this net (see Fig. 15).

Presented examples of dispersing and concentrating knots are the starts and finishes of event paths leading to and from these events.

Any of two events in event net with single distinguished starting event $Z_{\mathrm{A}}$ and single distinguished finishing event $Z_{B}$, where the events $Z_{k}, Z_{1} \neq Z_{A}, Z_{B}$, create the upper $\mathrm{U}$ and lower $\mathrm{L}$ alternative $\mathrm{D}$ and synchronous $\mathrm{C}$ event relation classes.

These classes are marked as following:

- upper alternative UD,

- upper synchronous UC,

- lower alternative LD,

- lower synchronous LC. 

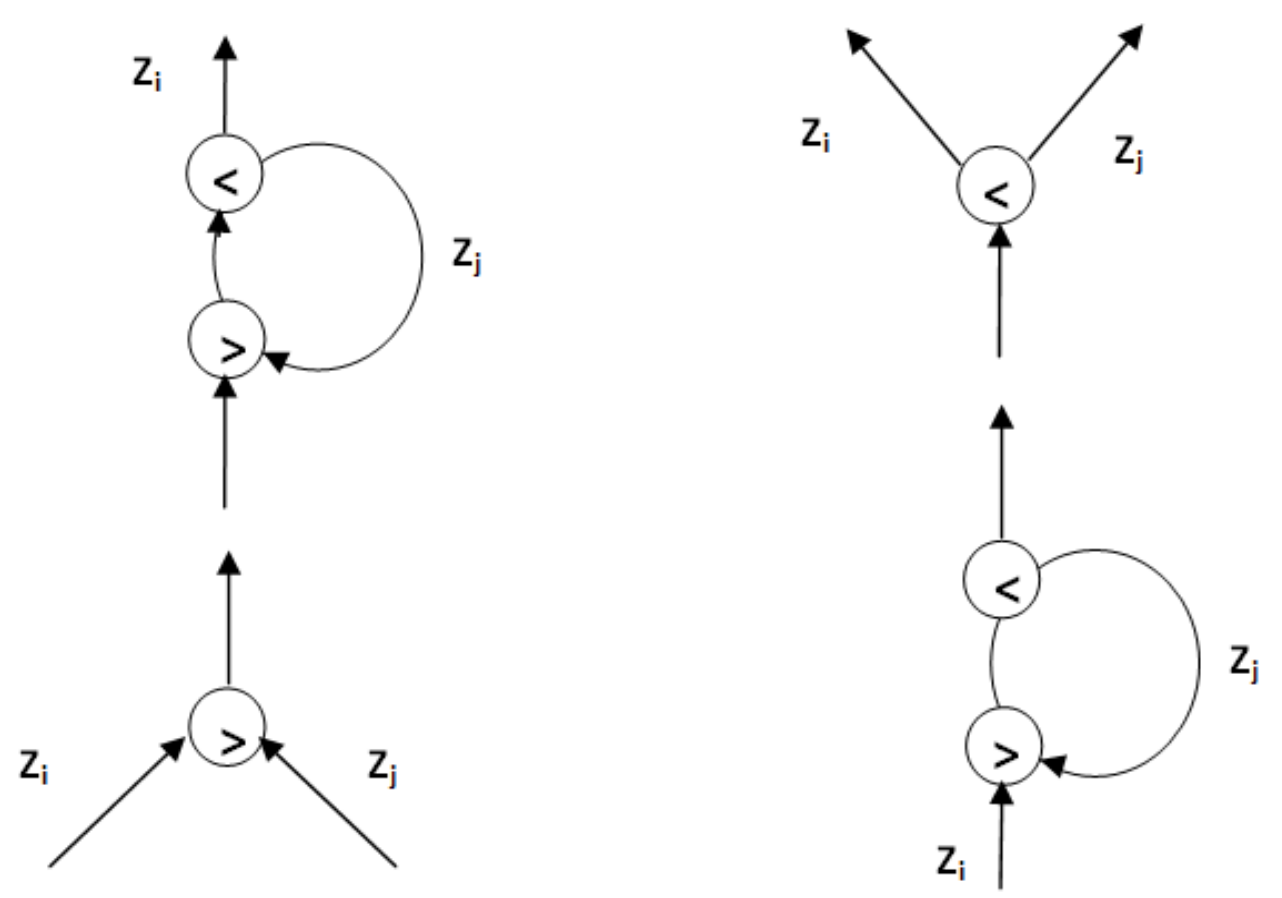

Figure 15. Four special cases, of concentrating $>$ and dispersing $<$ knots and events related with them

Two events $Z_{k}, Z_{1}$ of event net, which do not belong to the same series event sequence, belong to the upper alternative UD if paths from at least one dispersing alternative knot lead to them.

Two events $Z_{k}, Z_{1}$ of event net, which do not belong to the same series event sequence, belong to the upper synchronous UC if paths from at least one dispersing synchronous knot lead to them.

Two events $Z_{k}, Z_{1}$ of event net, which do not belong to the same series event sequence, belong to the lower alternative LD if paths from at least one concentrating alternative knot lead to them.

Two events $Z_{k}, Z_{1}$ of event net, which do not belong to the same series event sequence, belong to the lower synchronous LC if paths from at least one concentrating synchronous knot lead to them.

\section{$8 \quad$ Forbidden and obligatory graph figures - structural conditions of event net correctness}

Condition of event net structural correctness is the situation when any pair of events $Z_{k}, Z_{1}$ of this net belongs at the same time to the upper alternative UD and lower alternative LD class or only to upper synchronous UC and lower synchronous LC.

Forbidden graph figures in the event net are the violation of structural correctness conditions of the event net.

Figure 16 presents two forbidden graph figures (nonallowable) for the correctly constructed event net, which includes alternative and synchronous events.

Obligatory graph figures of event nets are the realization of structural correctness conditions of the event net.

Fig. 17 presents two obligatory graph figures (necessary) for the correctly constructed event net, which includes alternative and synchronous events.

Presented analysis indicate that the correctness conditions of event nets can be formulated in many ways, although the determination of topological properties of the net and its possible subnets is always a starting point. These properties can be used as modeling and controlling tools of discrete processes.

In some cases (see 9) the forbidden graph figures, as illustrated in Fig. 18, can be highly severe limitation for the net. 

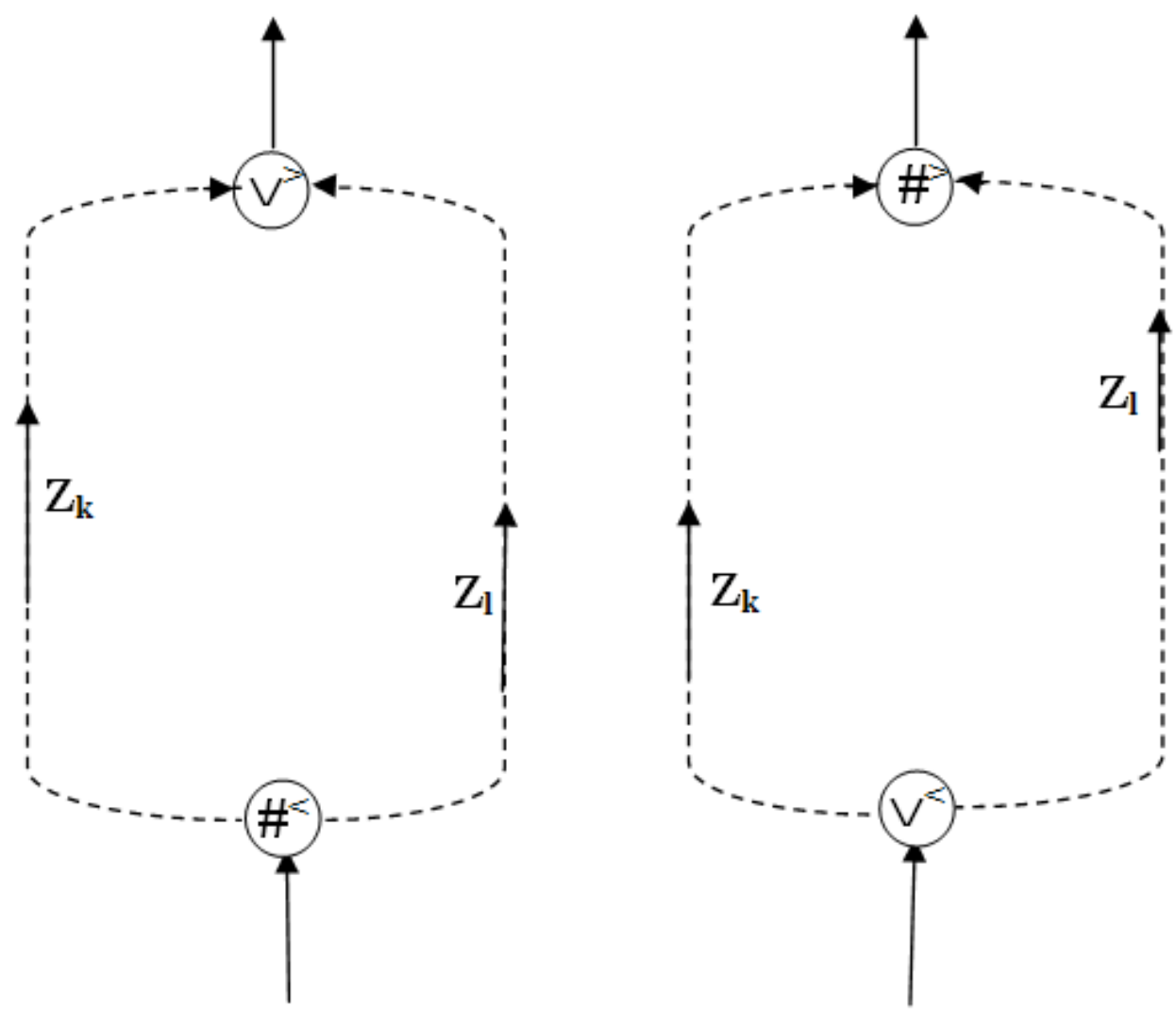

Figure 16. Forbidden graph figures in event net
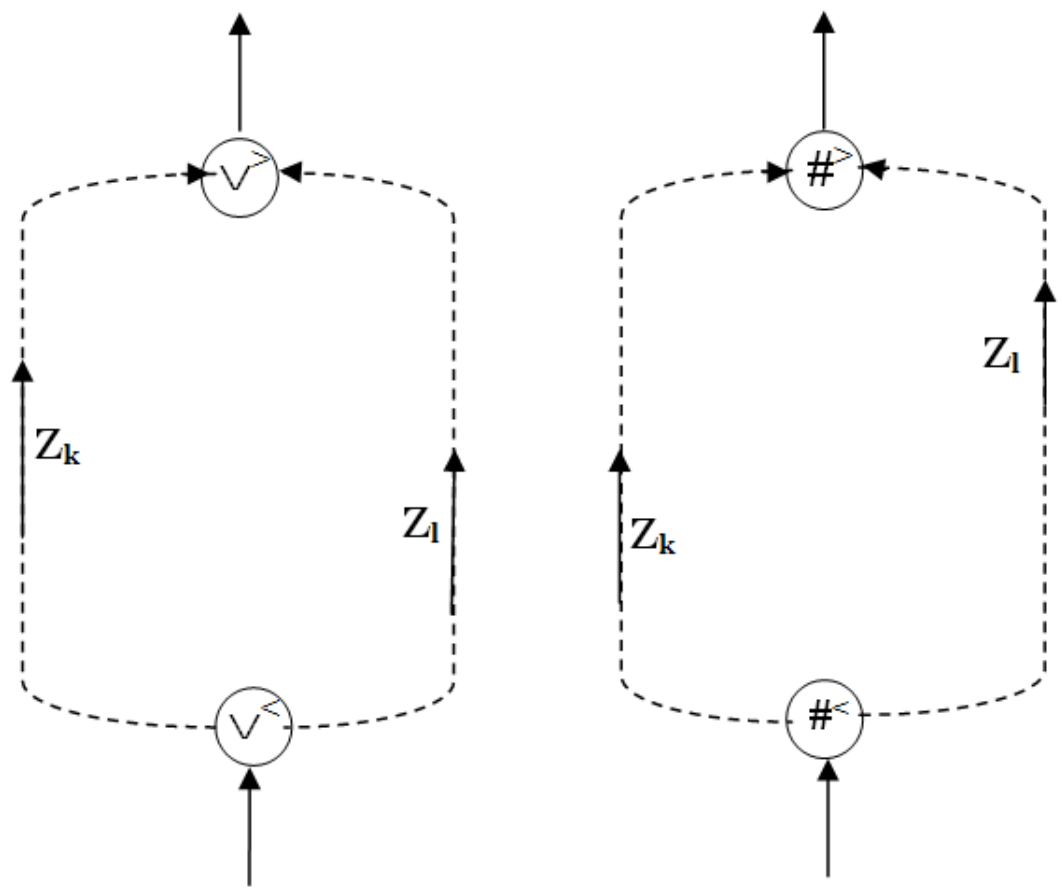

Figure 17. Obligatory graph figures in event net 


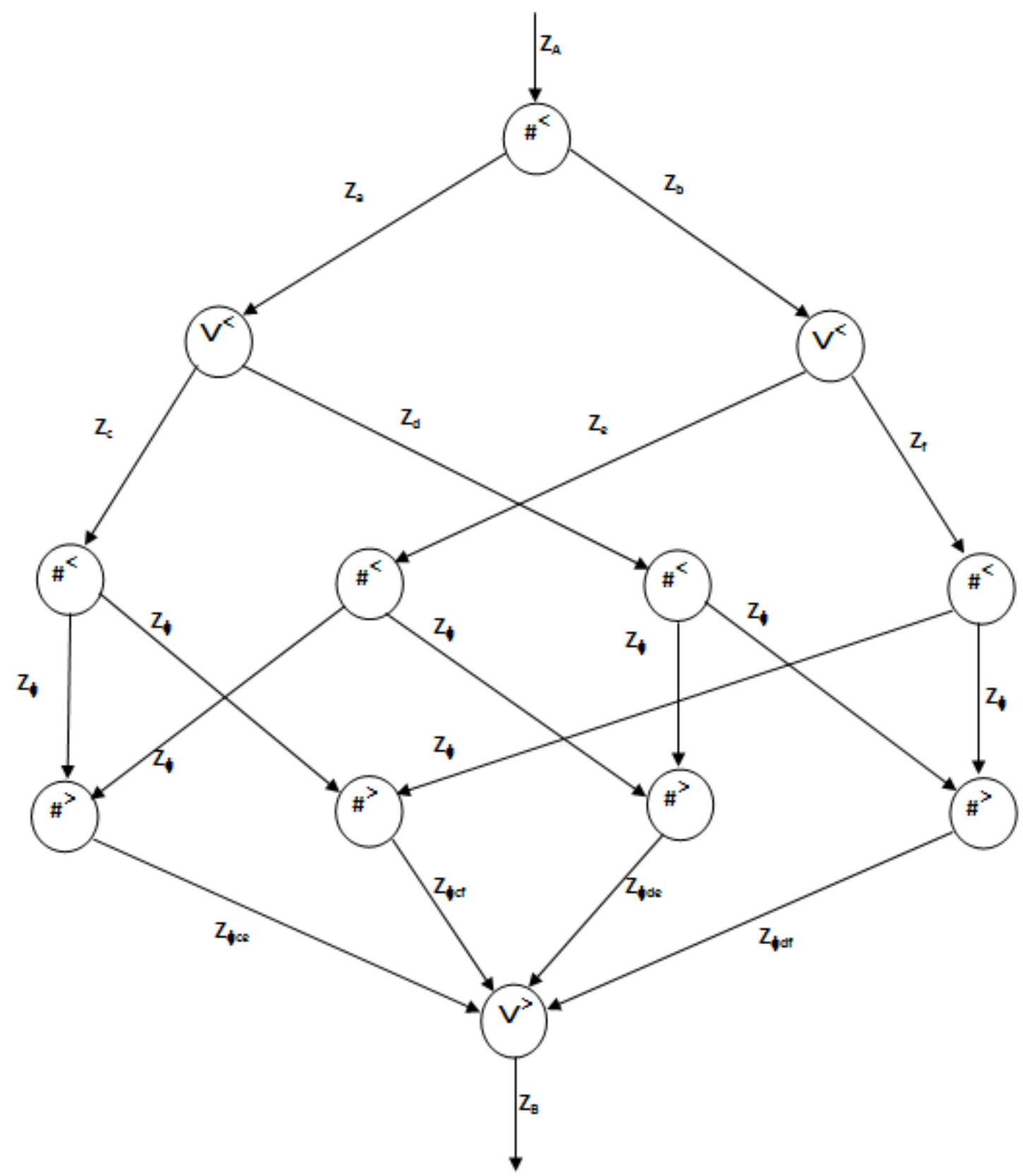

Figure 18. Example of event net that does not fulfill the correctness conditions expressed in the obligatory and forbidden graph figures

\section{Continuations}

It is easy to deduct that the net presented in Fig. 18 contains both obligatory and forbidden graph figures and according to p. 8 it does not fulfill the structural conditions of net correctness. Simultaneously detailed analysis of any of the four event pairs

$$
<\mathrm{Z}_{\mathrm{c}}, \mathrm{Z}_{\mathrm{e}}>,<\mathrm{Z}_{\mathrm{c}}, \mathrm{Z}_{\mathrm{f}}>,<\mathrm{Z}_{\mathrm{d}}, \mathrm{Z}_{\mathrm{e}}>,<\mathrm{Z}_{\mathrm{d}}, \mathrm{Z}_{\mathrm{f}}>
$$

indicates that it is manageable and therefore the $Z_{B}$ finishing event is manageable. In this case we speak of four event pairs from which only one can be realized as a result of preceding synchronous execution of the two $Z_{\mathrm{a}}, Z_{\mathrm{b}}$ events. 
However, if the analyzed event net (see Fig. 18) does not structurally guarantee the execution of at least one of the listed event pairs, than the $Z_{B}$ finishing event also would not be executed, what means the violation of net correctness conditions mentioned in point 8 .

Presented abalysis indicates that the event net correctness conditions can be formulated in many ways, although the determination of topological properties of the net and its possible subnets is always a starting point. These properties can be used as modeling and tools of correct designing of organizational structure.

Research on discrete event nets have their obvious implications and modeling languages, such as Perti nets, transforming nets, event algebra and many more.

The key is to apply such local functional limitations, which fulfilled by the net would assure its global functioning correctness - independent of process flow conditions. In other words, event net is a program (space) of its work and the event flow process is the execution of this program - if one uses an IT metaphor. The main success condition is the theoretical event net correctness that does not have to be proven or validated through tests. In research on this phenomenon the technical feasibility of the event is not crucial for the deliberations.

\section{$10 \quad$ References}

[1] Bromirski J. - Teoria automatów. WNT, Warszawa 1970.

[2] Gorbaov V.A. - Semanticeskaja teorija projektirovanija avtomatov. Izd. Energia, Moskva 1979.

[3] Krupa T. - Kolorowana sieć Petri'ego i sieć transformujac [in] Zeszyty Naukowe IOSP PW Organizacja i Zarządzanie Przemysłem. No. 15, Oficyna Wydawnicza PW, Warszawa 2002, pp. 5-24.

[4] Krupa T. - Sieciowe modele procesów zdarzeń [w] Komputerowo zintegrowane zarządzanie (ed. Knosala R.). Oficyna Wydawnicza PTZP, Opole 2006, pp. 55-66.

[5] Krupa T. - Elementy organizacji. Zasoby i zadania. WNT, Warszawa 2006. 